\title{
The mediating role of psychological need frustration on the relationship between frustration intolerance and existential loneliness
}

\author{
Mehmet Saricali ${ }^{1}$ D $\cdot$ Deniz Guler $^{2}$ (D)
}

Accepted: 2 February 2022 / Published online: 19 February 2022

(c) The Author(s), under exclusive licence to Springer Science+Business Media, LLC, part of Springer Nature 2022

\begin{abstract}
Although existential loneliness seems to be a natural consequence of being human, some people may experience it more intensely. In this study, it was aimed to investigate whether frustration intolerance, which is one of the basic concepts of Rational Emotive Behavior Therapy and psychological need frustration, which is the basic concept of Self-determination Theory predicted existential loneliness or not. A total of 294 adults were included in the study. The results showed that existential loneliness was directly predicted by frustration intolerance. As a result of the mediation test, all dimensions of psychological need frustration (autonomy frustration, relatedness frustration, and competence frustration) fully mediated the relationship between frustration intolerance and existential loneliness. The place of these findings in the literature was discussed and some recommendations were made.
\end{abstract}

Keywords Psychological need frustration $\cdot$ Frustration intolerance $\cdot$ Existential loneliness $\cdot$ Mediation analyses

The interrelatedness between self and others is the basic tenet of existential phenomenology (Sköld \& Roald, 2020). The ontological and epistemological consciousness of human beings expands through relationships and internalized relationships (Greening, 1992). In existential phenomenology, however, the impossibility of perfect communication between two people, uncertainty, and awareness of mortality salience means that all people can experience some degree of existential loneliness (Mayers \& Svartberg, 2001; Yalom, 1980).

Existential loneliness is a universally ontological human characteristic beyond the materialistic loss or inadequacy of intimate relationships (Bekhet et al., 2008). Although existential loneliness is an inherent feature of human beings, competitive consumerism and unstable relationships in the

Deniz Guler

denizguler@anadolu.edu.tr

Mehmet Saricali

mehmetsaricali@nevsehir.edu.tr

1 Faculty of Arts and Sciences, Department of Psychology, Nevşehir Hacı Bektaş Veli University, Nevşehir, Turkey

2 Faculty of Education, Department of Counseling and Guidance, Anadolu University, Eskişehir, Turkey post-modern period produce a fertile and vulnerable environment for existential loneliness (Bound Alberti, 2018). In the USA, for example, there has been a growing concern for existential loneliness (Funch, 2021).

The paradox of the need for relationality and the inability to fully capture the feelings and experiences between two subjects constitute the basic loneliness, which is one of the main determinants of existential anxiety (Bruggen et al., 2015). In addition to existential anxiety, the relationship was found between high depression, anxiety, and high existential isolation scale (Pinel et al., 2017), which is used to measure existential loneliness (Constantino et al., 2019). In a study conducted with young adults in Turkey, positive relation among existential loneliness among depression, hopelessness, suicidal ideations; negative relations among existential loneliness and meaning in life, perceived social support, and optimism was reported (Gökdemir-Bulut \& Bozo, 2018). Existential loneliness has basic cognitive and emotional determinants. Schemas for relationships and the persons themselves may be considered among the basic elements that may trigger existential loneliness. Therefore, this study hypothesized that frustration intolerance may be one of the main predictors of existential loneliness. 


\section{Frustration Intolerance}

Frustration intolerance describes the rigid demands for gratification and comfort as well as the vulnerability of the ego depending on social approval and perfect well-being (Harrington, 2005). Based on Rational Emotive Behavior Therapy (REBT), frustration intolerance is shaped around the belief that external reality will never be tolerated if it does not fully comply with personal demands (Harrington, 2011). Frustration intolerance-related beliefs are mostly self-imposed (DiGiuseppe, 2011). The rigidity or flexibility of this kind of belief is the main determinant of low/high frustration intolerance (Dryden \& David, 2008).

In REBT, frustration intolerance is considered to be the underlying cause of many psychological difficulties (Harrington, 2006). Setting strict and high cognitive standards is one of the prominent causes of dysfunctional outcomes. In a study conducted with this philosophy, the mediating role of frustration intolerance in the relationship between maladaptive perfectionism and dysphoria was demonstrated (Stanković et al., 2015). A similar line of reasoning, the mediating role of frustration intolerance in the relationship between maternal control and school learned helplessness was revealed (Filippello et al., 2018). Rigid personal standards may also trigger avoidance behavior. For example, in a study, a positive relationship was found between discomfort intolerance and procrastination (Uzun-Ozer et al., 2012). Subsequent research showed that high frustration tolerance is associated with high well-being (Efstathiou, 2013). In another study, the relationship of four subscales of frustration intolerance with maladaptive results was examined in a clinical sample. Results demonstrated that anger was predicted by entitlement, anxiety by emotional intolerance, and depressive mood by discomfort intolerance (Stanković \& Vukosavljević-Gvozden, 2011). In a non-clinical sample also, it was reported that there was a high correlation among emotional intolerances subscales and anxiety as well as depression, among discomfort intolerance entitlement sub-scales and anger (Filippello et al., 2014).

Frustration intolerance can emerge through meta-schemas formed in relationships. Frustration intolerance is also an important determinant of the psychological need frustration in existential and relational dialogue. Therefore, this study has aimed to examine the mediating role of psychological need frustration in the relationship between frustration intolerance and existential loneliness.

\section{Psychological Need Frustration}

Psychological need satisfaction and frustration is a basic motivation model philosophically put forward within the scope of Self-determination Theory (SDT) based on the propositions of the humanistic theory. In SDT, it is argued that whereas one has a growth motivation under supportive conditions and may develop vulnerability in a controlling and critical environment (Deci \& Ryan, 1985; Ryan \& Deci, 2000; Vansteenkiste \& Ryan, 2013)., it has been empirically demonstrated that in SDT, instead of dichotomous separation of motivation as autonomy and heteronomy human motivation can continue with different combinations of these two motivations (Ryan \& Deci, 2006). The fulfillment or frustration of three basic psychological needs which are autonomy, competence, and relatedness, as well as the shift from controlled to autonomy within the continuum of motivation is considered a key determinant of psychological functioning (Deci \& Ryan, 2008).

Psychological need satisfaction and frustration are not mutually exclusive phenomena. In other words, low psychological need satisfaction does not mean high need frustration (Davis \& Turner, 2020). Accordingly, for example, need satisfaction and frustration were found to uniquely predict well-being (Heissel et al., 2018) and depressive symptomology (Chen et al., 2015; Nishimura \& Suzuki, 2016). Further, need frustration was found to uniquely predict poor sleep quality in patients with chronic fatigue (Campbell et al., 2018), depressive and physical symptoms in athletes (Bartholomew et al., 2011), relatedness frustration predicted negative affect in the children (Schmidt et al., 2020). Based on given findings, it was expected in this study that need frustration can predict uniquely existential loneliness in cultures where relative relationships are intertwined.

\section{The Current Study}

Existential loneliness is generally an area of underresearch and has been rarely explored in cultures where the collective self-construal predominates (Chung et al., 2020; Park \& Pinel, 2020). To the best of our knowledge, this is the first research to discuss that existential loneliness can emerge as a result of basic psychological need frustration originated from frustration intolerance related cognitions. it was considered that Autonomy frustration is related to sense of pressure, Relatedness frustration sense of social alienation and competence frustration sense of ineffectiveness, failure as well as helplessness (Vansteenkiste et al., 2020). Considering the above definition, in cultures with a greater sense of collective self, rejection in relationships, perceptions of power and boundaries are crucial in terms of existential loneliness. Although a study found a high sense of collective self-construal and low perception of existential isolation (Park \& Pinel, 2020), the main argument of this research is that frustration of basic psychological needs is a main explanatory factor for existential loneliness, even in cultures where collective 
self-construal is relatively high. In situations where social control is high, which may be relatively high in collective cultures, authenticity is considered crucial in terms of basic psychological need satisfaction (Ryan \& Ryan, 2019). People with a high perception of existential loneliness may also feel inauthentic and indecisive (Long et al., 2021). Accordingly, high perfectionism and high need frustration (Boone et al., 2014), as well as low frustration intolerance (Stanković et al., 2015), and high frustration intolerance and high flourishing (Ruiz-Ortega et al., 2021) were found to be correlated. Hence, frustration intolerance, which conceptualizes perfectionist and vulnerable cognitions towards relationships and achievement, is expected to predict need frustration.

Theoretically, emphasis on self-awareness, unconditional acceptance of the self, and confrontation without distorting external reality in RET overlap with existential approaches (Ward, 2011). Existential loneliness is a construct with cognitive and affective components, which occurs as a result of the individual's realization their own finitude (Ettema et al., 2010). With the awareness of finitude, frustration intolerance, a fundamental irrational belief, may appear, consistent with the basic premises of RET. Moreover, relationships, one of the basic concepts of existential well-being (Hoffman et al., 2015) may be adversely effected by frustration intolerance-related beliefs. Frustration intolerance may positively effect need frustration that is shaped by relationships. At the same time, it may increase existential loneliness through need frustration. Arguably, frustration intolerance-based cognition may damage relationships by being the focus of psychological needs. The need for competence, autonomy and relatedness which are the main determinants of living a meaningful as well as psychologically rich life (Oishi \& Westgate, 2021) may also trigger existential loneliness. As a consequence of intra-fusion, excessive dependence on relationships and the pursuit of constant affirmation for others may bust existential loneliness (Davidov et al., 2021).

Although existential loneliness is an inevitable condition for an entity conscious of finitude, it has been stated that there is a very limited number of researches on this subject (Helm, 2019). In addition, it was considered that the fear of Covid-19 may confound effect in terms of existential loneliness, as the Covid-19 pandemic causes mortality to be more salience and strict rules are applied to restrict interpersonal contact in the pandemic. Mortality-based anxiety is the main reason behind existential loneliness e.g. (van Tilburg, 2021). Moreover, although physical loneliness is low, it is theoretically suggested that existential and emotional loneliness may be experienced more deeply in socially embedded cultures in the case of rigid relational and social norms and the perception of deviation from these norms (Heu et al., 2021). Therefore, in this under-research area, testing the following hypotheses was found worth investigating:
Hypothesis 1: Frustration intolerance predicts existential loneliness directly.

Hypothesis 2: Frustration intolerance predicts existential loneliness through autonomy frustration which is the dimension of psychological need frustration.

Hypothesis 3: Frustration intolerance predicts existential loneliness through competence frustration which is the dimension of psychological need frustration.

Hypothesis 4: Frustration intolerance predicts existential loneliness through relatedness frustration which is the dimension of psychological need frustration.

\section{Method}

This descriptive study was conducted cross-sectional with online volunteer participants. A mediation test was applied within the scope of the research purpose. Considering the possible confounding effect of the current covid-19 pandemic on existential loneliness, the fear of Covid-19 and the age were included in the mediation model as a covariate.

\section{Participants and Procedure}

The research was carried out with volunteer Turkish adults using online data collection methods. All stages of the study were reviewed by the Nevşehir Hacı Bektaş Veli University Scientific Research and Ethical Board (Protocol Number: E.9753, Date: 14.05.2020). The online questionnaire link arranged was delivered to the participants by the researchers using social media and e-mail groups. Participants first read the informed consent form and pointed out that they voluntarily participated in the further parts. A total of 313 people participated in the study. In the preliminary analyzes, 19 outliers data $\left(\chi_{(4)}^{2}=18.47, p=.001\right)$ were excluded and the final analyzes were carried out with the remaining 294 participants. Hence, the participants of the study consisted of 294 (175 females, 119 males) aged between 18 and 65 $(M=29.32, S D=7.65)$.

\section{Measures}

\section{The Frustration Discomfort Scale}

The Frustration Discomfort Scale (FDS) is a five-point Likert-type scale developed by Harrington (2005) to determine the level of frustration intolerance. FDS was adapted to Turkish by Uzun-Ozer et al. (2012). Turkish FDS consists of 24 items with four subscales: Discomfort intolerance (e.g. "I need the easiest way around a problem; I can't stand making a hard time of it.", Emotional intolerance (e.g. "I can't bear to feel that I am losing my mind"), Achievement intolerance (e.g. 'I can't stand being prevented from achieving my full 
potential'), and Entitlement intolerance (e.g. I can't stand it if people act against my wishes"). The scale is scored between 24 and 120, and high scores indicate low frustration intolerance. The internal consistency coefficient of the scale was calculated as .86, and the test-retest coefficient as .70. In the present study, Cronbach's alpha coefficient was determined as .94 .

\section{Basic Psychological Needs Satisfaction and Frustration Scale}

Basic Psychological Needs Satisfaction and Frustration Scale (BPNSFS) was developed to assess psychological need satisfaction as well as need frustration (Chen et al., 2015). The scale was adapted to Turkish by Selvi and Bozo (2020). The scale consists of 24 items and six subscales. Three of the subscales (Autonomy Satisfaction, Competence Satisfaction, and Relatedness Satisfaction) target the level of satisfaction of psychological needs, the other three are [Autonomy Frustration (e.g. "I feel pressured to do too many things"), Competence Frustration (e.g. "Ifeel insecure about my abilities"), and Relatedness Frustration (e.g. "I feel excluded from the group I want to belong to")] measure the level of frustration. Three subscales measuring psychological need frustration were used in this study. Cronbach's alpha internal consistency coefficients of the scale dimensions were obtained as .74 for autonomy frustration, .79 for competence frustration, and .84 for relatedness frustration. In the present study, internal consistency coefficients were calculated as $.77, .76$, and .79 , respectively.

\section{Existential Loneliness Questionnaire}

Existential Loneliness Questionnaire (ELQ) is a six-point Likert type developed to assess existential loneliness (Mayers et al., 2002). Although the questionnaire was originally developed with HIV-infected participants, the Turkish adaptation study was carried out in a large non-clinical sample (Gökdemir-Bulut \& Bozo, 2018). The Turkish version consists of 20 items and three subscales: Loneliness in Social Ties (e.g. "I have had trouble finding people I can talk to"), Loneliness in Close Relationships (e.g. "If I had the right relationship, I would never feel alone"), and Finding Meaning in Life. (e.g. "The universe is full of meaning"). The ELQ is scored between 20 and 120, and high scores indicate a high perception of existential loneliness. Cronbach's alpha internal consistency coefficients were calculated as .80 for finding meaning in life, .85 for loneliness in social ties, and .70 for loneliness in close relationships. In the current study, Cronbach's alpha coefficients were $.82, .79$, and .85 for, respectively, and .92 for the total scale.

\section{The Fear of COVID-19 Scale}

Fear of Covid-19 scale (FCVS-19) is developed to assess individuals' fears associated with COVID-19 disease (Ahorsu et al., 2020). The scale consists of seven items (e.g. "It makes me uncomfortable to think about COVID19 ") which were answered using ranging from 1 ("strongly disagree") to 5 ("strongly agree"). In this study, the Turkish version of FCVS-19 (Satici et al., 2020) was used. In the adaptation study of the FCVS-19, CFA analyses showed that the scale had a good fit to the data $\left(\chi_{(13, \mathrm{~N}=1304)}^{2}=299.47\right.$, $p<.05 ; \mathrm{GFI}=.936 ; \mathrm{SRMR}=.061 ; \mathrm{NFI}=.912 ; \mathrm{CFI}=.915)$. The scores to be obtained from the scale can vary between 7 and 35. The increasing scores indicate high fears caused by Kovid-19. Cronbach's alpha coefficient for the FCVS-19 was .85 in the Turkish adaptation study (Satici et al., 2020), and it was .88 in the current study.

\section{Data Analysis}

Mediation analysis was performed using PROCESS version 3.5 as an SPSS macro developed by Hayes (2018). In this analysis, Model 4 (Hayes, 2018, p. 149, 585) which can be used to test the mediation of two or more variables from parallel multi-mediation models were used. Since the data is collected online, there is no need for missing data analysis because there is no missing value. Before this regressionbased mediation analysis, prerequisite values were examined. Before further analyses, data were also reviewed for multivariate normality, linearity, and multicollinearity.

In this study, regarding normality assumptions skewness values were between -.52 and .83 as well as kurtosis values were between -.46 and .74 . Since these coefficients are between \pm 2 , the data were normally distributed (George \& Mallery, 2010). Considering the multi-collinearity assumptions, a correlative link of over .80 was not observed between any variables (see, Table 1). Since VIF values (between 1.32 and 2.21) are less than 10 (James et al., 2013) and tolerance values (between .45 and .76) are greater than .10 (Tabachnick \& Fidell, 2001) indicated that there is no multicollinearity problem.

\section{Results}

\section{Relationships between Variables and Descriptive Statistics}

Before examining direct and indirect relationships, correlation values between three variables as well as descriptive results were examined. These values are shown in Table 1 .

As a result of the correlation analysis, it was found that there was a positive relationship between all variables 
Table 1 Descriptive statistics and Pearson correlation coefficients for the variables $(n=294)$

\begin{tabular}{lllllllll}
\hline & 1 & 2 & 3 & 4 & 5 & 6 & 7 & 8 \\
\hline 1. Gender & - & & & & & & \\
2. Age & .08 & - & & & & & \\
3. Frustration Intolerance & -.11 & -09 & - & & & & \\
4. Autonomy Frustration & -.09 & -.05 & $.47^{* *}$ & - & & & \\
5. Competence Frustration & -.04 & $-.23^{* *}$ & $.38^{* *}$ & $.60^{* *}$ & - & & & \\
6. Relatedness Frustration & $.15^{*}$ & -.11 & $.23^{* *}$ & $.46^{* *}$ & $.65^{* *}$ & - & & \\
7. Existential Loneliness & .10 & $-.19^{* *}$ & $.30^{* *}$ & $.48^{* *}$ & $.61^{* *}$ & $.68^{* *}$ & - & \\
8. Fear of COVID-19 & $-.30^{* *}$ & .07 & $.42^{* *}$ & $.27^{* *}$ & $.22^{* *}$ & $.17^{* *}$ & $.16^{* *}$ & - \\
$M$ & .41 & 29.32 & .80 & 11.86 & 9.5 & 8.5 & 47.36 & 17.79 \\
$S D$ & .49 & 7.65 & 17.91 & 3.56 & 3.34 & 3.22 & 17.24 & 5.99 \\
\hline${ }^{*} p<.05,{ }^{* *} p<.01$ & & & & & & & &
\end{tabular}

$(p<.05)$. Before mediation analysis, the predictive power of frustration intolerance on existential loneliness was examined (see, Table 1). The results showed that $12 \%$ of existential loneliness was explained by frustration intolerance $(\beta=.26)$.

\section{Results of Mediation Analysis}

In the mediation analysis, it was examined whether the psychological need frustration mediated the relationship between frustration intolerance and existential loneliness by controlling age and fear of COVID-19. The autonomy frustration, competence frustration, and relatedness frustration, which are three sub-dimensions of psychological need frustration, were included in the model separately. Results for this analysis are shown in Fig. 1.

When the direct effects of variables are examined, it was observed that frustration intolerance predicted autonomy frustration $(\beta=.43, p<.001)$, relatedness frustration $(\beta=.18$, $p<.01)$, and competence frustration $(\beta=.32, p<.001)$ positively. Beside, existential loneliness was positively predicted by autonomy frustration $(\beta=.12, p<.05)$, relatedness frustration $(\beta=.47, p<.001)$, and competence frustration $(\beta=.19, p<.01)$. Finally, when the mediator variables included the model, the direct effect of frustration intolerance on existential loneliness became insignificant $(c=.26$, $\left.p<.001, c^{\prime}=.06, p>.05\right)$. Therefore, it can be inferred from these results that autonomy frustration, relatedness frustration, and competence frustration are fully mediate relations between frustration intolerance and existential loneliness (see, Hayes, 2018, p. 461). The results regarding whether the full mediation effect is significant are shown in Table 2.

Bootstrap results showed that the indirect effects of autonomy frustration on the relationship between frustration intolerance and existential loneliness are significant $(X \rightarrow M 1 \rightarrow Y=.050, \% 95 \mathrm{CI}=.002, .101)$. Similarly, the indirect effects of relatedness frustration on the relationship between frustration intolerance and existential loneliness

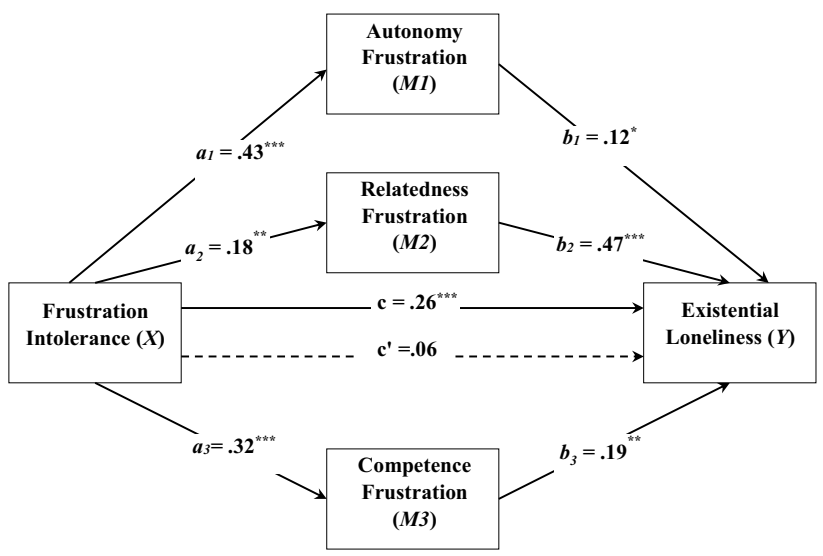

Fig. 1 Parallel multiple mediation model for the direct and indirect effects of frustration intolerance on existentinal loneliness through autonomy frustration, relatedness frustration, and competence frustration, controling age and fear of COVID-19. ${ }^{*} p<.05{ }^{* *} p<.01$ ${ }^{* * * *} p<.01$

are significant $(X \rightarrow M 2 \rightarrow Y=.084, \% 95 \mathrm{CI}=.031, .144)$. Finally, the indirect effects of competence frustration on the relationship between frustration intolerance and existential loneliness are also significant $(X \rightarrow M 3 \rightarrow Y=.061$, $\% 95 \mathrm{CI}=.019, .112)$. In addition, it was determined that all variables in the model together explain $52 \%$ of the existential loneliness. Consequently, need frustration had a full mediating effect on the relationship between frustration intolerance and existential loneliness.

\section{Discussion}

In this study, it was aimed to explore whether frustration intolerance predicts existential loneliness through psychological need frustration. As a result of the research, existential loneliness was significantly predicted by frustration 
Table 2 Parameters and 95\% CIs for the paths of the mediation model

\begin{tabular}{|c|c|c|c|c|}
\hline \multirow[t]{2}{*}{ Indirect Effects } & \multirow[t]{2}{*}{ Bootstrap Coeff } & \multirow[t]{2}{*}{ SE } & \multicolumn{2}{|c|}{$\% 95 \mathrm{CI}$} \\
\hline & & & Lower & Upper \\
\hline Frustration Intolerance $(X) \rightarrow$ Autonomy Frustration $(M 1) \rightarrow$ Existential Loneliness $(Y)$ & .050 & .025 & .002 & .101 \\
\hline Frustration Intolerance $(X) \rightarrow$ Relatedness Frustration $(M 2) \rightarrow$ Existential Loneliness $(Y)$ & .084 & .029 & .031 & .144 \\
\hline Frustration Intolerance $(X) \rightarrow$ Competence Frustration $(M 3) \rightarrow$ Existential Loneliness $(Y)$ & .061 & .024 & .019 & .112 \\
\hline
\end{tabular}

SE Standard Error; $C I$ Confidence Interval

intolerance, and psychological need frustration fully mediated this relationship.

The result of this research indicated that frustration intolerance is a significant predictor of existential loneliness. This finding relatively contradicts the view that the experience resulting from uncertainty in the existential process is largely determined as a result of intuitive and insightful evaluations rather than rational views (van den Bos, 2009). Accordingly, although existential loneliness is arguably intuitively and emotionally felt, frustration intolerance-related beliefs may also shut the self into its own imprisonment. In other words, in line with the views of the REBT, existential loneliness which may occur intuitively and emotionally may arise from dogmatic demands for oneself, success and relationships. The setting of rigid standards for the self is also theoretically considered as the main trigger of alienation (Horney, 1992). What is more, the prediction of frustration intolerance to existential loneliness in this study indirectly coincides with the studies revealing the relationship between low frustration intolerance and high depressive symptoms (Filippello et al., 2014). Although it is theoretically argued that, the cognitive-behavioral perspective may be insufficient in existential issues, however, in a meta-analysis study examining interventions for death anxiety, it was revealed that the most effective therapeutic intervention was the cognitivebehavioral approach (Menzies et al., 2018). Considering the above finding and this finding that frustration intolerance predicts existential loneliness together, it can be argued that the Rational Emotional Therapy approach may be effective in existential issues.

Another finding of the study is that autonomy, competence, and relatedness frustration which are the dimensions of psychological need frustrations were mediators in the relationship between frustration intolerance and existential loneliness. The finding regarding mediating roles of autonomy frustration, in this study, as in the same direction with the research finding that autonomy frustration is an important determinant of depressive symptoms in both individualistic and collective cultures (Chen et al., 2015). This finding for the relatedness frustration coincides with the theoretical proposition that existential loneliness can occur as in consequence of the lack of relational encounters (Yalom, 1980). It can be speculated that the way competence is mediated, one can experience a sense of control through relationships, and this can reduce existential loneliness. Further, in this context, it is argued that frustration intolerance can be triple in the elimination of problems beyond the passive acceptance of external reality (Harrington, 2011). Therefore, the finding in this study, which suggests the relationship between high psychological need frustration and high frustration intolerance, is in line with the research finding that irrational beliefs are an important determinant of self-determination motivation in athletes (Davis \& Turner, 2020).

\section{Conclusion}

As a result of the research, it was revealed that frustration intolerance and need frustration are significant predictors of existential loneliness, independent of the fear of COVID19. This finding shed light on the fact that the propositions of self-determination theory and rational emotional therapy may together form a metatheory for coping with existential loneliness. The findings of this study relatively support the argument that cognitive-behavioral approaches should include more existential issues and integrate with existential approaches (Heidenreich et al., 2021). Therefore, this study has an important result in terms of filling a gap that classical cognitive-behavioral therapy has in understanding human nature.

\section{Limitations and Recommendations}

Firstly, although structured quantitative methods may be criticized in obtaining knowledge in existential approaches a mediation test has been performed in this study within the scope of methodological pluralism. In further inquiries on existential loneliness, questions based on phenomenological and grounded theory can contribute to the portray of inclusive knowledge.

Secondly, in this research, although the diversity of the participants and the sampling adequacy according to the purpose are relatively achieved through the online data collection method, existential loneliness can be investigated by using different sampling methods from a wider population in future studies. Given that, existential loneliness can be 
perceived intuitively as much as a cognitive assessment, the experiential sampling method can reveal important findings in this regard.

Third, this research was conducted descriptively and cross-sectionally. In future research, in order to examine the formation, development as well as coping with existential loneliness, encounter group studies and mixed research design supported by longitudinal qualitative inquiry can reveal seminal findings.

Data Availability The datasets generated during and/or analyzed during the current study are available from the authors on reasonable request.

\section{Declarations}

Ethical Statement All procedures conducted were approved by the Nevşehir Hacı Bektaş Veli University Scientific Research and Ethical Board (Protocol Number: E.9753, Date: 14.05.2020).

Informed Consent Inform Informed consent was obtained from all participants included in the study.

Conflict of Interest It has been reported by the authors that there is no conflict of interest.

\section{References}

Ahorsu, D. K., Lin, C. Y., Imani, V., Saffari, M., Griffiths, M. D., \& Pakpour, A. H. (2020). The fear of COVID-19 scale: Development and initial validation. International Journal of Mental Health and Addiction. Advance online publication. https://doi.org/10.1007/ s11469-020-00270-8

Bartholomew, K. J., Ntoumanis, N., Ryan, R. M., Bosch, J. A., \& Thøgersen-Ntoumani, C. (2011). Self-determination theory and diminished functioning: The role of interpersonal control and psychological need thwarting. Personality and Social Psychology Bulletin, 37(11), 1459-1473. https://doi.org/10.1177/01461 67211413125

Bekhet, A. K., Zauszniewski, J. A., \& Nakhla, W. E. (2008). Loneliness: A concept analysis. Nursing Forum (Hillsdale), 43(4), 207-213. https://doi.org/10.1111/j.1744-6198.2008.00114.x

Boone, L., Vansteenkiste, M., Soenens, B., Van der Kaap-Deeder, J., \& Verstuyf, J. (2014). Self-critical perfectionism and binge eating symptoms: A longitudinal test of the intervening role of psychological need frustration. Journal of Counseling Psychology, 61(3), 363-373. https://doi.org/10.1037/a0036418

Bound Alberti, F. (2018). This "modern epidemic": Loneliness as an emotion cluster and a neglected subject in the history of emotions. Emotion Review, 10(3), 242-254. https://doi.org/10.1177/17540 73918768876

Bruggen, V. V., Vos, J., Westerhof, G. J., Bohlmeijer, E. T., \& Glas, G. (2015). Systematic review of existential anxiety instruments. Journal of Humanistic Psychology, 55(2), 173-201. https://doi. org/10.1177/0022167814542048

Campbell, R., Vansteenkiste, M., Delesie, L., Tobback, E., Mariman, A., Vogelaers, D., \& Mouratidis, A. (2018). Reciprocal associations between daily need-based experiences, energy, and sleep in chronic fatigue syndrome. Health Psychology, 37(12), 1168-1178. https://doi.org/10.1037/hea0000621

Chen, B., Vansteenkiste, M., Beyers, W., Boone, L., Deci, E. L., Van der Kaap-Deeder, J., Lens, W., Matos, L., Mouratidis, A., Ryan, R. M., Sheldon, K. M., Soenens, B., Petegem, S. V., \& Verstuyf, J. (2015). Basic psychological need satisfaction, need frustration, and need strength across four cultures. Motivation and Emotion, 39(2), 216-236. https://doi.org/10.1007/ s11031-014-9450-1

Chung, B. P. M., Olofsson, J., Wong, F. K. Y., \& Rämgård, M. (2020). Overcoming existential loneliness: A cross-cultural study. BMC Geriatrics, 20(1), 347-347. https://doi.org/10.1186/ s12877-020-01753-y

Constantino, M. J., Sommer, R. K., Goodwin, B. J., Coyne, A. E., \& Pinel, E. C. (2019). Existential isolation as a correlate of clinical distress, beliefs about psychotherapy, and experiences with mental health treatment. Journal of Psychotherapy Integration, 29(4), 389-399. https://doi.org/10.1037/int0000172

Davidov, J., Russo-Netzer, P. J. A., \& Stress, \& Coping. (2021). Exploring the phenomenological structure of existential anxiety as lived through transformative life experiences. Anxiety, Stress, and Coping, 1-16. https://doi.org/10.1080/10615806.2021.1921162

Davis, H., \& Turner, M. J. (2020). The use of rational emotive behavior therapy (REBT) to increase the self-determined motivation and psychological well-being of triathletes. Sport Exercise and Performance Psychology, 9(4), 489-505. https://doi.org/10.1037/ spy0000191

Deci, E. L., \& Ryan, R. M. (1985). Intrinsic motivation and self-determination in human behavior. Springer.

Deci, E. L., \& Ryan, R. M. (2008). Self-determination theory: A macrotheory of human motivation, development, and health. Canadian Psychology/Psychologie Canadienne, 49(3), 182-185. https://doi.org/10.1037/a0012801

DiGiuseppe, R. (2011). Reflection on my 32 years with Albert Ellis. Journal of Rational-Emotive \& Cognitive-Behavior Therapy, 29(4), 220-227. https://doi.org/10.1007/s10942-010-0118-9

Dryden, W., \& David, D. (2008). Rational emotive behavior therapy: Current status. Journal of Cognitive Psychotherapy, 22(3), 195209. https://doi.org/10.1891/0889-8391.22.3.195

Efstathiou, N. (2013). Self-control and well-being: The moderating roles of frustration intolerance and emotional intensity. (dissertation/thesis), ProQuest Dissertations Publishing.

Ettema, E. J., Derksen, L. D., \& Leeuwen, E. V. (2010). Existential loneliness and end-of-life care: A systematic review. Theoretical Medicine and Bioethics, 31(2), 141-169. https://doi.org/10.1007/ s11017-010-9141-1

Filippello, P., Harrington, N., Buzzai, C., Sorrenti, L., \& Costa, S. (2014). The relationship between frustration intolerance, unhealthy emotions, and assertive behaviour in Italian students. Journal of Rational-Emotive \& Cognitive-Behavior Therapy, 32(4), 257-278. https://doi.org/10.1007/s10942-014-0193-4

Filippello, P., Harrington, N., Costa, S., Buzzai, C., \& Sorrenti, L. (2018). Perceived parental psychological control and school learned helplessness: The role of frustration intolerance as a mediator factor. School Psychology International, 39(4), 360-377. https://doi.org/10.1177/0143034318775140

Funch, B. S. (2021). Art, emotion, and existential well-being. Journal of Theoretical and Philosophical Psychology, 41(1), 5-17. https:// doi.org/10.1037/teo0000151

George, D., \& Mallery, M. (2010). SPSS for windows step by step: A simple guide and reference, 17.0 update (10th ed.). Pearson.

Gökdemir-Bulut, B. P., \& Bozo, Ö. (2018). The psychometric validity and reliability of the Turkish version of the existential loneliness questionnaire. Current Psychology, 37(1), 401-413. https://doi. org/10.1007/s12144-016-9534-z 
Greening, T. (1992). Existential challenges and responses. The Humanistic Psychologist, 20(1), 111-115. https://doi.org/10.1080/08873 267.1992.9986784

Harrington, N. (2005). The frustration discomfort scale: Development and psychometric properties. Clinical Psychology \& Psychotherapy, 12(5), 374-387. https://doi.org/10.1002/cpp.465

Harrington, N. (2006). Frustration intolerance beliefs: Their relationship with depression, anxiety, and anger, in a clinical population. Cognitive Therapy and Research, 30(6), 699-709. https://doi.org/10.1007/ s10608-006-9061-6

Harrington, N. (2011). Frustration intolerance: Therapy issues and strategies. Journal of Rational-Emotive \& Cognitive-Behavior Therapy, 29(1), 4-16. https://doi.org/10.1007/s10942-011-0126-4

Hayes, A. F. (2018). Introduction to mediation, moderation, and conditional process analysis: A regression-based approach. Guilford Press, NY, USA.

Heidenreich, T., Noyon, A., Worrell, M., \& Menzies, R. (2021). Existential Approaches and Cognitive Behavior Therapy: Challenges and Potential. International Journal of Cognitive Therapy, 14(1), 209-234. https://doi.org/10.1007/s41811-020-00096-1.

Heissel, A., Pietrek, A., Flunger, B., Fydrich, T., Rapp, M. A., Heinzel, S., \& Vansteenkiste, M. (2018). The validation of the German basic psychological need satisfaction and frustration scale in the context of mental health. European Journal of Health Psychology, 25(4), 119-132. https://doi.org/10.1027/2512-8442/a000017

Helm, P. J. (2019). Towards a theory of interpersonal isolation: Adding existential isolation to the mix. (Doctoral Dissertation, The University of Arizona). The University of Arizona University Libraries. http://hdl.handle.net/10150/632994. Accessed 4 April 2021.

Heu, L. C., Hansen, N., Van Zomeren, M., Levy, A., Ivanova, T. T., Gangadhar, A., \& Radwan, M. J. P. R. (2021). Loneliness across cultures with different levels of social embeddedness: A qualitative study. Personal Relationships, 28(2), 379-405.

Hoffman, L., Vallejos, L., Cleare-Hoffman, H. P., \& Rubin, S. (2015). Emotion, relationship, and meaning as core existential practice: Evidence-based foundations. Journal of Contemporary Psychotherapy, 45(1), 11-20. https://doi.org/10.1007/s10879-014-9277-9

Horney, K. (1992). Our inner conflicts: A constructive theory of neurosis. W W Norton \& Co.

James, G., Witten, D., Hastie, T., \& Tibshirani, R. (2013). An introduction to statistical learning with applications in $R$. Springer.

Long, A. E., Pinel, E. C., Daily, J. R., \& Costello, A. E. (2021). Existential isolation and the struggle for belief validation. The British Journal of Social Psychology. https://doi.org/10.1111/bjso.12492

Mayers, A. M., \& Svartberg, M. (2001). Existential loneliness: A review of the concept, its psychosocial precipitants and psychotherapeutic implications for HIV-infected women. British Journal of Medical Psychology, 74(4), 539-553. https://doi.org/10.1348/000711201161082

Mayers, A. M., Khoo, S. T., \& Svartberg, M. (2002). The existential loneliness questionnaire: Background, development, and preliminary findings. Journal of Clinical Psychology, 58(9), 1183-1193. https:// doi.org/10.1002/jclp.10038

Menzies, R. E., Zuccala, M., Sharpe, L., \& Dar-Nimrod, I. J. J. o. a. d. (2018). The effects of psychosocial interventions on death anxiety: A meta-analysis and systematic review of randomised controlled trials. 59, 64-73.

Nishimura, T., \& Suzuki, T. (2016). Basic psychological need satisfaction and frustration in Japan: Controlling for the big five personality traits. Japanese Psychological Research, 58(4), 320-331. https://doi. org/10.1111/jpr.12131

Oishi, S., \& Westgate, E. C. (2021). A psychologically rich life: Beyond happiness and meaning. Psychological Review. Advance online publication. https://doi.org/10.1037/rev0000317
Park, Y. C., \& Pinel, E. C. (2020). Existential isolation and cultural orientation. Personality and Individual Differences, 159, 109891. https:// doi.org/10.1016/j.paid.2020.109891

Pinel, E. C., Long, A. E., Murdoch, E. Q., \& Helm, P. (2017). A prisoner of one's own mind: Identifying and understanding existential isolation. Personality and Individual Differences, 105, 54-63. https://doi. org/10.1016/j.paid.2016.09.024

Ruiz-Ortega, A. M., Álvarez, N. S., \& Martos, M. P. B. (2021). Chilean validation of the frustration discomfort scale: Relation between intolerance to frustration and discomfort and emotional intelligence. Current Psychology. https://doi.org/10.1007/s12144-021-02135-2

Ryan, R. M., \& Deci, E. L. (2000). Self-determination theory and the facilitation of intrinsic motivation, social development, and wellbeing. American Psychologist, 55(1), 68-78. https://doi.org/10. 1037/0003-066X.55.1.68

Ryan, R. M., \& Deci, E. L. (2006). Self-regulation and the problem of human autonomy: Does psychology need choice, self-determination, and will? Journal of Personality, 74(6), 1557-1586. https://doi.org/ 10.1111/j.1467-6494.2006.00420.x

Ryan, W. S., \& Ryan, R. M. (2019). Toward a social psychology of authenticity: Exploring within-person variation in autonomy, congruence, and genuineness using self-determination theory. Review of General Psychology, 23(1), 99-112. https://doi.org/10.1037/gpr00 00162

Satici, B., Gocet-Tekin, E., Deniz, M. E., \& Satici, S. A. (2020). Adaptation of the fear of COVID-19 scale: Its association with psychological distress and life satisfaction in Turkey. International Journal of Mental Health and Addiction. Advanced publication online. https:// doi.org/10.1007/s11469-020-00294-0

Schmidt, A., Neubauer, A. B., Dirk, J., \& Schmiedek, F. (2020). The bright and the dark side of peer relationships: Differential effects of relatedness satisfaction and frustration at school on affective wellbeing in children's daily lives. Developmental Psychology, 56(8), 1532-1546. https://doi.org/10.1037/dev0000997

Selvi, K., \& Bozo, Ö. (2020). The dark side of bodybuilding: The role of bodybuilding activities in compensation of frustrated basic psychological needs. Motivation and Emotion, 1-19. https://doi.org/10. 1007/s11031-019-09805-6

Sköld, A. B., \& Roald, T. (2020). An existential structure of love. The Humanistic Psychologist. https://doi.org/10.1037/hum0000165

Stanković, S., \& Vukosavljević-Gvozden, T. (2011). The relationship of a measure of frustration intolerance with emotional dysfunction in a student sample. Journal of Rational-Emotive \& Cognitive-Behavior Therapy, 29(1), 17-34. https://doi.org/10.1007/s10942-011-0128-2

Stanković, S., Matić, M., Vukosavljević-Gvozden, T., \& Opačić, G. (2015). Frustration intolerance and unconditional self-acceptance as mediators of the relationship between perfectionism and depression. Psihologija, 48(2), 101-117. https://doi.org/10.2298/PSI1502101S

Tabachnick, B. G., \& Fidell, L. S. (2001). Using multivariate statistics (4th ed.). Allyn and Bacon.

Uzun-Ozer, B., Demir, A., \& Harrington, N. (2012). Psychometric properties of frustration discomfort scale in a Turkish sample. Psychological Reports, 111(1), 117-128. https://doi.org/10.2466/08.02.18. PR0.111.4.117-128.

van den Bos, K. (2009). Making sense of life: The existential self trying to deal with personal uncertainty. Psychological Inquiry, 20(4), 197-217. https://doi.org/10.1080/10478400903333411

van Tilburg, T. G. (2021). Social, emotional, and existential loneliness: A test of the multidimensional concept. The Gerontologist, 61(7), e335-e344.

Vansteenkiste, M., \& Ryan, R. M. (2013). On psychological growth and vulnerability: Basic psychological need satisfaction and need 
frustration as a unifying principle. Journal of Psychotherapy Integration, 23(3), 263.

Vansteenkiste, M., Ryan, R. M., \& Soenens, B. (2020). Basic psychological need theory: Advancements, critical themes, and future directions. Motivation and Emotion, 44(1), 1-31. https://doi.org/10.1007/ s11031-019-09818-1

Ward, J. J. (2011). "oh, the humanity!": Kurt Vonnegut and rational emotive behavior Therapy's existential rejoinder to the irrationality of the human condition. The Humanistic Psychologist, 39(2), 105-120. https://doi.org/10.1080/08873267.2011. 540151

Yalom, I. D. (1980). Existential psychotherapy. Basic Books.

Publisher's Note Springer Nature remains neutral with regard to jurisdictional claims in published maps and institutional affiliations. 